\title{
Report from the seminar on the past, present, and future of tobacco control, Harvard University Department of the History of Science, Cambridge, Massachusetts, October 2016'
}

\author{
Mateusz Zatoński ${ }^{1,2}$, Michał Stokłosa ${ }^{3}$ \\ 'London School of Hygiene and Tropical Medicine, London, UK \\ ${ }^{2}$ Health Promotion Foundation, Nadarzyn, Poland \\ ${ }^{3}$ American Cancer Society, Atlanta, GA, USA
}

ADDRESS FOR CORRESPONDENCE: Mateusz Zatoński, London School of Hygiene and Tropical Medicine, Keppel Street, London WC1E 7HT, UK, e-mail: mateusz.zatonski@lshtm.ac.uk

\section{INTRODUCTION}

The roundtable seminar on the past, present, and future of tobacco control took place in October 2016 at the Harvard University Science Center. It was hosted by Professor Allan Brandt and the Harvard University Department of the History of Science, and co-organised by the Polish Health Promotion Foundation (Fundacja "Promocja Zdrowia"). The event brought together a range of international public health leaders and scholars, including health economists, political scientists, historians of science, health advocates, and policymakers. During the seminar the extensive range of regional and disciplinary expertise of the guests was drawn upon to generate broad, strategic thinking on the history and perspectives of tobacco control, as well as future research and collaboration ideas. The main goals of the meetings were:

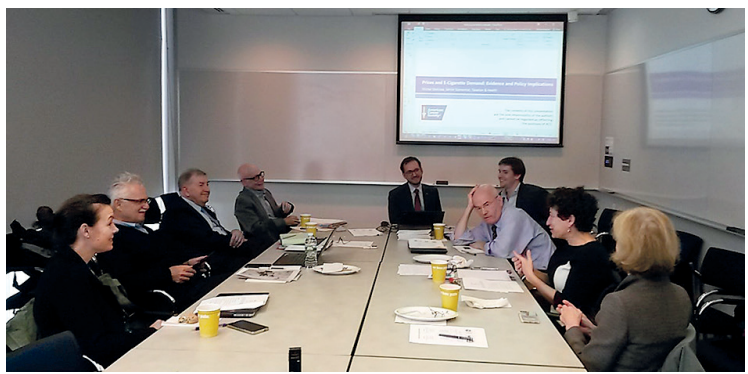

Photo credits: Aleksandra Herbeć

FIG. 1. From left to right: Gina Kruse, Rajmund Dąbrowski, Witold Zatoński, Allan Brandt, Michał Stokłosa, Mateusz Zatoński, Gregory Connolly, Naomi Oreskes, Nancy Rigotti
1. To identify the key learning opportunities emerging from historical research for global tobacco control leaders and health advocates.

2. To appraise the current challenges to effective tobacco control efforts globally.

3. To initiate collaboration on a roadmap for the future of global tobacco control.

\section{HARM REDUCTION APPROACHES TO TOBACCO CONTROL}

Historically, harm reduction in tobacco control was a relatively minor issue. Apart from nicotine replacement therapies (NRT) and some forms of smokeless tobacco, no nicotine-containing product had the potential to reduce the harm of tobacco use. This changed with the introduction of electronic nicotine delivery systems (ENDS) into the marketplace in the mid-2000s. As these products - the most popular to date is the electronic or e-cigarette - have become increasingly prevalent, it is clear that we must address the central issues raised by their exponential growth in the marketplace. A historical aversion towards harm reduction approaches exists within a significant segment of the public health community in the United States (US) [1]. Seminar participants were asked to consider whether harm reduction approaches are more favoured by tobacco control advocates in other parts of the world, and, if so, why this is the case.

Allan Brandt noted that while we often talk about a Euro-American-Australian consensus on tobacco control and positive changes in tobacco-related behaviours 
in these parts of the world, the fact is that the cultures of tobacco control remain very different between these regions. For example, the differences between the United Kingdom (UK) and the US in terms of their traditions of harm reduction, which have been reflected in the much stricter regulation of e-cigarettes in the US. Another example of these differences is the minimum age of use of alcohol, which in the US is set at 21 years, while in most of Europe it is 18 or lower. Brandt also brought up the recent change in the laws regulating cannabis in several US states [2], observing the irony that marijuana laws are being liberalised at the same time as access to tobacco products is becoming more restrictive, for example with around 100 communities in Massachusetts raising the age of purchase of tobacco products from 18 to 21 [3]. Those "finer notions of culture and history", according to Brandt, should be kept in mind "as potential guides to developing some consensus about the future of tobacco control".

Naomi Oreskes emphasised the importance of acknowledging the different priorities given to harm reduction depending not only on geographic region, but also on disciplinary background. For clinicians who have an obligation to their individual patients, a harm reduction approach can be much more acceptable than to public health lawyers or historians. This sentiment was echoed by Nancy Rigotti, who said that while in her role as a smoking cessation clinician she would recommend tried-and-tested cessation drugs to patients struggling to quit in the first place, if this did not work, she would be happy to see them completely switch over to harm reducing products, such as e-cigarettes, rather than continue smoking traditional cigarettes.

One of the problems with harm reduction in tobacco control, in Allan Brandt's opinion, is that its definition is set in relation to the world's most dangerous legal product - the traditional cigarette. From the point of view of the tobacco industry this creates an opportunity whereby any product they create, regardless its addictiveness, will be viewed as progress by a large part of the public health community. "So in a way", Brandt concluded, "we've let the regulatory standard be set by incredibly dangerous combustible products".

\section{ELECTRONIC NICOTINE DELIVERY SYSTEMS}

E-cigarettes and next generation nicotine delivery systems have become one of the symbols of the debate over the merits and disadvantages of harm reduction approaches. Many harm reduction proponents believe that ENDS can play a role in tobacco control that could be similar to that played by methadone therapies in evidence-based heroin control policies - helping some users quit and substantially reducing harm among others who for various reasons are unable or not willing to overcome their addiction, and for whom, in the absence of these harm reduction mechanisms, there would be a high probability of continued use of a much more hazardous substance [4].

With the advent and popularity of e-cigarettes, one of the looming challenges for health advocacy today is the question of their regulation. The debate over these products reprises many themes that have recurred throughout the history of tobacco control - such as the tension between harm reduction approaches and the precautionary principle, or the challenges posed by disruptive innovation to the public health status quo as technological dissemination outstrips the knowledge context. The experts were posed the question of whether there is an appropriate regulatory framework for dealing with products which are inherently dangerous, but the moderate use of which might be preferable to the alternative.

While the science on the effectiveness of e-cigarettes as cessation aids is still far from complete, the few existing studies suggest that they are not a silver bullet, with similar quitting rates to nicotine replacement therapy [5]. However, as Nancy Rigotti observed, it is quite clear that they are significantly less harmful than combustible tobacco products. Thus, a temporary switch from cigarettes to e-cigarettes, followed by quitting both, would be the ideal scenario for their use as cessation tools. However, Rigotti continued, "what we are now seeing is that currently most people are doing what's called dual-use, they're using both cigarette and e-cigarettes. If they're doing that then is that really harm reduction?". Research shows that reducing the consumption of combustible tobacco by a little does not yield significant health benefits [6]. In this context people engaging in the dual-use of e-cigarettes and combustible tobacco might be misguided into thinking they are helping themselves reduce harm, while in fact they are not achieving this goal in any meaningful way.

With combustible cigarettes discredited, in Naomi Oreskes' opinion, the focus on e-cigarettes is a strategic decision of the tobacco industry. With the reputation of being significantly less harmful than traditional smoking, their modern design, little if any second-hand smoke, and an appeal to children, they can be profitable, especially if their relatively high price is taken into account. Allan Brandt and Nancy Rigotti suggested that the industry will most likely not displace combustibles, as they remain fantastically profitable. Instead it will address different products to different markets. E-cigarettes will be sold to those who have the access and resources to utilise them, while combustible cigarettes will continue to dominate among poorer groups and in the developing world. The effect of this will be an increase in health disparities between low-and high-income populations.

However, Greg Connolly pointed out that the most popular e-cigarettes are yet to turn a profit after being on the market for two years, while the profit margin for a pack of Marlboro, despite all the existing anti-smok- 
ing policies, remains as high as $40 \%$. In his opinion, big tobacco companies such as Philip Morris will not pursue the e-cigarette route, as they realise that this product is an inefficient nicotine delivery mechanism. Instead, he suggested that the real public health concern should lie with next generation products, such as the Marlboro IQOS, or the R.J. Reynolds VUSE FOB. These products are the first step towards combining drug delivery with iPhone-like capacity and internet connectivity, as well as digital marketing tools. These might allow to customise the delivery of nicotine by monitoring usage patterns and adjusting the dose and frequency.

Companies such as Philip Morris have invested billions of dollars into next generation products, marketing them as improved tools of harm reduction [7]. The pharmaceutical industry is far from matching this commitment with the much lower sums, in the hundreds of millions, it has spent on cessation therapies and medications. The tobacco industry is further helped by the weak regulation of high technology nicotine products, which stands in contrast to the strict rules governing the introduction of any new cessation drugs. Greg Connolly concluded by expressing concern that the failure to adequately regulate the conventional market might mean being "forced to allow through [...] a highly abusive product on the market which gives us slight risk reduction, but also a loss of autonomy, loss of control [...] This is removing self-administration, you are no longer in control of what goes into your body, you're relinquishing that, so you're losing autonomy under this technology, as many people are to their iPhone. [...] It's sort of the best of the two worlds, it's the Marlboro man combined with Steve Jobs".

\section{TREATMENT OF TOBACCO DEPENDENCE}

Effective smoking cessation support remains the most direct way of controlling the burden of tobacco-related illness and death. Despite e-cigarettes having captured a large portion of the public debate on quitting aids in recent years, various other, better researched treatment methods exist and are still not adequately used. Access to such treatment varies widely across and within countries, as do the treatments themselves [8]. Seminar participants explored the historical development and current status of treatment methods, as well as their strengths and limitations.

Nancy Rigotti gave an overview of the field of smoking cessation. She pointed out that while offering help to quit is part of the WHO Framework Convention for Tobacco Control MPOWER package of technical measures and resources, it is often viewed as the "forgotten piece of tobacco control", as it is given less attention than other tobacco control measures, such as smoking bans or taxation. However, from the perspective of clinical medicine, getting current smokers to quit it is not only the most cost-effective method, but also the one where pos-

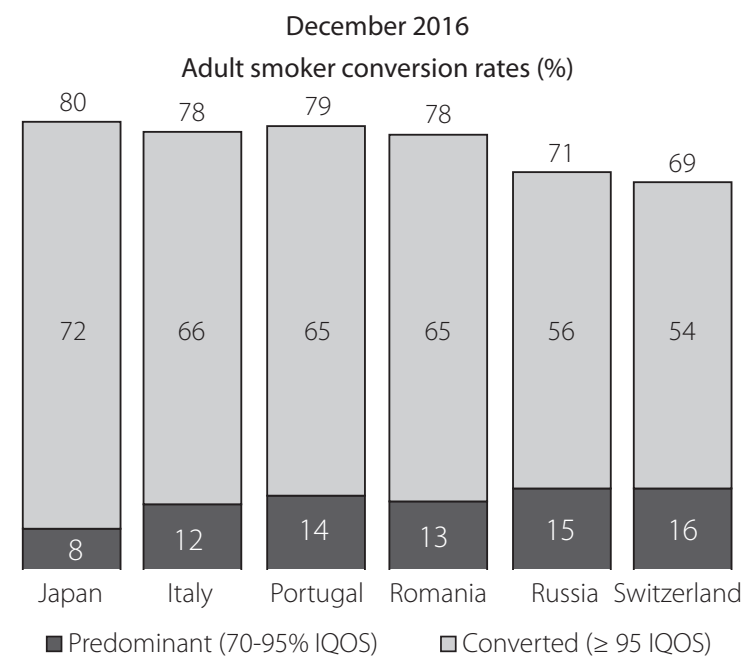

Source: Philip Morris Japan/Italy/Portugal/Romania/Russia/Switzerland IQOS User Panels.

FIG. 2. IQOS conversion rates for smokers in test markets

itive health effects and reduced deaths can be observed most quickly. While most smokers want to quit, a point emphasised by Witold Zatoński, few succeed long-term as a result of any single quit attempt. Nancy Rigotti presented data showing that only $6 \%$ of those who try to quit succeed in doing so without any help, while $20-30 \%$ do so with the best treatments available - nonetheless, even a brief intervention by a physician improves chances of quitting. However, only one-third of those trying to quit use any treatment at all. The big challenge of cessation today is therefore delivering treatments to more smokers.

Since smoking is partly a behavioural issue, and partly an addiction, cessation methods must tackle both. While counselling and psychosocial support can help with breaking the habit, pharmacotherapy is intended to assist in overcoming the addiction. In a country like the US available methods include telephone quitlines, NRT, Buproprion and Varenicline. While studies have shown that Varenicline is the most effective cessation drug, it got a bad name due to concerns about its psychiatric side-effects which, according to Nancy Rigotti, reduced the willingness of doctors to prescribe it and of patients to take it. Despite studies showing that these concerns were largely unfounded [9], Varenicline's reputation might have suffered irreversibly due to bad press. Witold Zatoński talked about cytisine, an alkaloid used in the production of smoking cessation drugs such as Tabex and Desmoxan. The first cytisine-based drugs were developed already in the 1960s. Its effectiveness is comparable to Varenicline, while its price is much lower as it is a generic agent [10]. However, cytisine remains largely unavailable outside Central and Eastern Europe. Meanwhile, it has achieved great commercial success in Poland, where after heavy advertising campaigns in the 
last few years sales grew from a few thousand packages per year to almost one million. Witold Zatoński pointed out the irony in the fact that cytisine, despite being a safe, effective, and natural smoking cessation pharmaceutical with a proven track record, is still available only in a handful of states, while synthetic e-cigarettes, with little science supporting their use as cessation tools, are accessible in many countries around the world.

In concluding the section on the treatment of tobacco dependence, participants discussed the role of healthcare professionals in improving the delivery of existing treatments to smokers. Most people in Europe and North America come in contact with a doctor or a nurse during any given year, providing an opportunity for what Nancy Rigotti referred to as a "teachable moment". The illness that brings people to see a medical professional is an additional motivation to quit. In addition, the meeting is also a chance for the doctor to issue a prescription for smoking cessation drugs. One way of getting doctors more engaged in effectively using this "teachable moment" is by beginning to think about tobacco use as a chronic disease. Instead of asking patients if they are ready to quit, treatment should be offered to them auto- matically, as in the case of other chronic diseases such as diabetes. Gina Kruse noted that chronic disease management is increasingly moving towards a systems-based approach and that perhaps this is also a path smoking cessation should take. Rather than rely solely on the individual doctor's counsel to their patient, ways should be sought to automatise the process.

\section{TOBACCO TAXATION}

Increasing the price of cigarettes has been shown to be one of the most effective ways of reducing overall smoking prevalence [11]. However, while smoking rates have generally declined in many high-income countries, they remain persistently high among people from the lowest socio-economic groups [12]. The seminar discussion touched upon the role of taxation and the optimal strategies in which it should be used to tackle smoking while not becoming a disproportionate burden on the poor.

The discussion was opened by Michał Stokłosa’s point that the relationship between tobacco taxes and tobacco consumption is remarkably straightforward - as the taxes go up, tobacco products become less afforda-

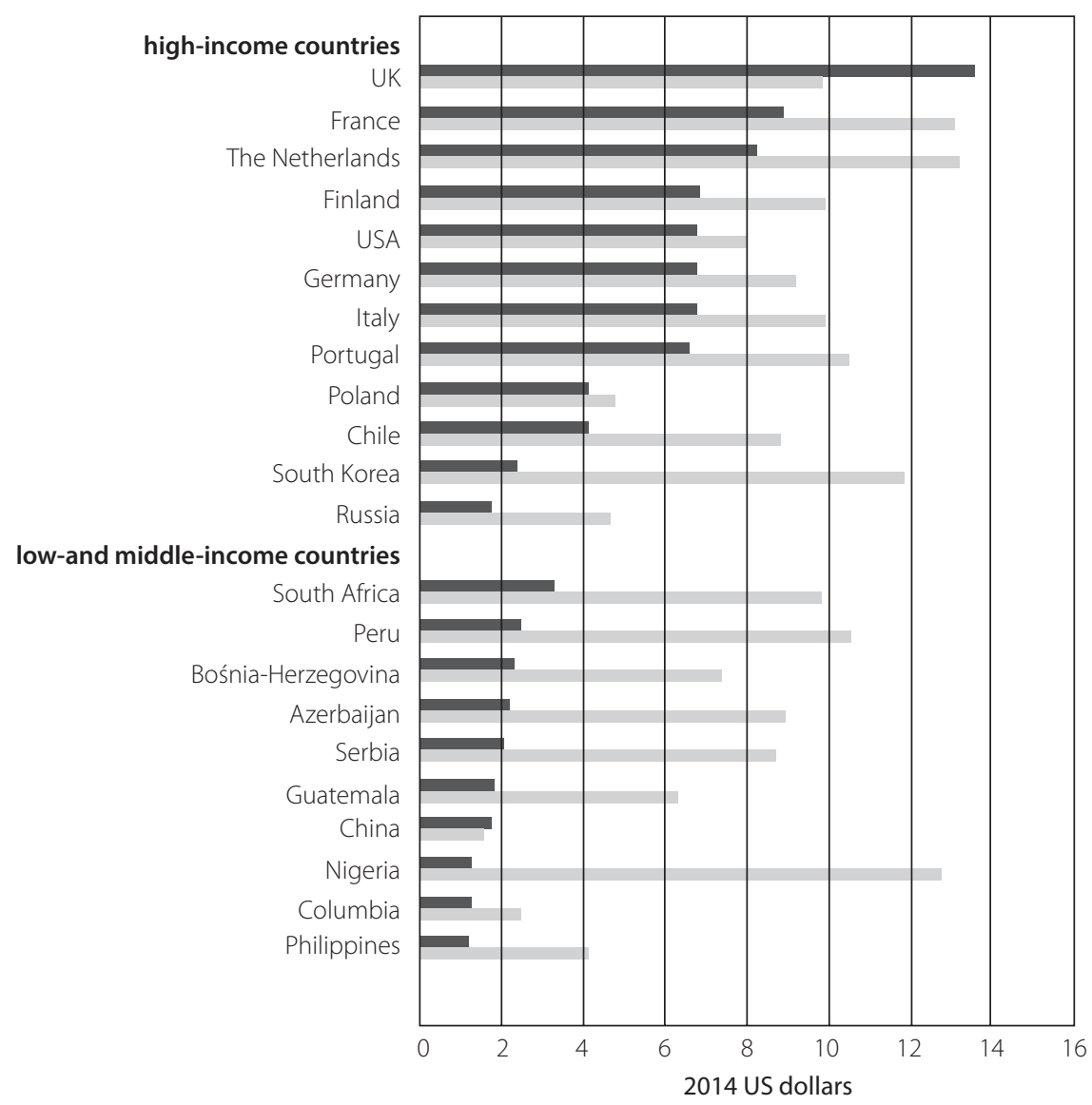

$\square$ Combustible cigarette $\quad \square$ Disposable e-cigarette

Source: Liber AC, Drope JM, Stoklosa M. Combustible cigarettes cost less to use than e-cigarettes: Global evidence and tax policy implications. Tob Control 2017; 26(2): 158-163.

FIG. 3. Prices of conventional cigarettes and disposable e-cigarettes in different countries 
ble, and their consumption decreases. European countries with low tobacco taxes, such as Greece, tend to have higher consumption levels than countries with high tobacco taxes, such as the UK. In addition, people from lower socio-economic groups, as well as young people, are more price-responsive to changes in taxation. Allan Brandt added that taxation is one of the few tobacco control measures that seems to be universal and work irrespective of the culture or state in which it is implemented. Therefore, it should hold a prominent position in any tobacco control toolkit. He also expressed the hope that higher taxes could become an important tributary to public health funding and tobacco control. Michał Stokłosa was sceptical about this possibility, pointing out that governments tend to be unwilling to restrain their ability to spend tax money, and would rather keep it in one budget rather than earmark it for a particular public health objective.

Michał Stokłosa also outlined findings suggesting that e-cigarettes are more price-elastic than traditional cigarettes, and that there is a substitution effect between the two products - when the prices of cigarettes went up, some smokers moved to e-cigarettes. As a result, if a harm reduction approach is to be taken, it would be advisable to incentivise current smokers of combustible cigarettes to switch to e-cigarettes by making the former less affordable.

Some of the criticisms of the taxation approach were assessed by the participants. One of them, mentioned by Mateusz Zatoński, was the tobacco industry argument claiming that tax increases lead to an increase in smuggling and the sale of illegal cigarettes. Allan Brandt mentioned the critique that tobacco taxes are regressive and exacerbate income inequalities. Michał Stokłosa rebuked the first argument, citing research showing that the proportion of smuggled cigarettes in the European Union is not significantly increasing despite tax increases, and remains around $14 \%$ of the entire cigarette market which incidentally means that as the overall cigarette market declines, so does the absolute number of illicit cigarettes. As to the second critique, it is a real challenge, as smoking rates remain higher among people of lower socio-economic status, and it is those who carry on smoking who will indeed carry a greater part of the tax burden. In order to address this, the entire system would need to become more progressive, for instance by covering smoking cessation drugs with health insurance in order to lessen the burden on the poor.

\section{PAST SUCCESSES IN TOBACCO CONTROL}

Using any metric, whether it be smoking prevalence, the absolute number of smokers, or the incidence of smoking-related diseases, tobacco control across large parts of Europe and North America has been very successful in the last decades [13]. The participants of the seminar where asked to reflect on what lessons can be

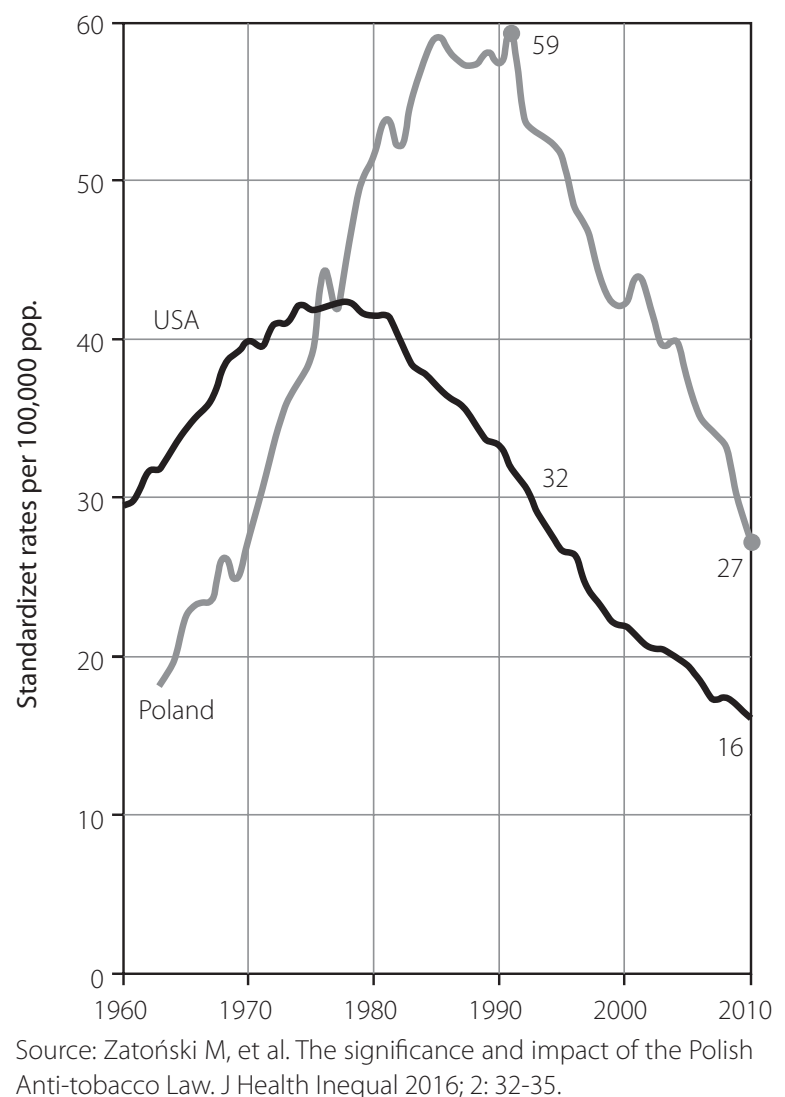

FIG. 4. Lung cancer, males, 35-54, Poland vs. USA

drawn from these past achievements in order to inform future attempts at dealing with the tobacco epidemic.

Allan Brandt noted that in the last decades several countries, including the US, have provided positive models for global tobacco control, implementing tobacco advocacy and policy precedents that were then replicated in other countries. In the Polish case, explored by Witold Zatoński, this example has been supplemented by direct support from the American and British tobacco control community. One example of this support was the Conference on A Tobacco Free New Europe, held in the town of Kazimierz, in Poland in November 1990, just one year after the collapse of the Berlin Wall. The conference was organized by the American Cancer Society and the International Union Against Cancer. It was attended by public health leaders, such as Richard Peto and Greg Connolly, and served as an opportunity to train a new generation of Central and East European anti-tobacco advocates, as well as to structure the tobacco control efforts in the region in the next years. The Kazimierz Declaration, which concluded the meeting, and which was based on the gold standards of tobacco control developed in the West, formed the basis of the tobacco control roadmap implemented in Poland in the 1990s. It also became a tool for anti-tobacco advocates lobbying the Polish government, a process which in 1995 culminated with the passage of the most comprehensive tobacco control law in the region [14]. 


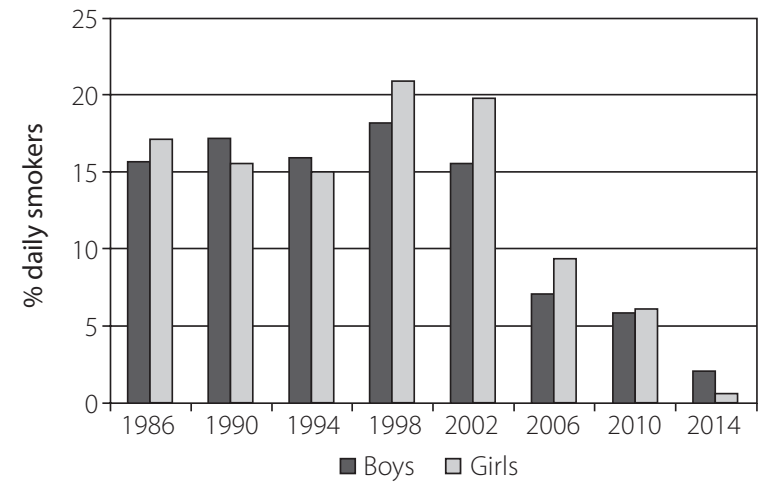

Source: Aaro LE, et al. Trends in smoking among Polish and Norwegian youth 1986-2014. J Health Inequal 2016; 2: 44-51.

FIG. 5. Proportion of daily smokers among 15 year olds by gender and year, Norway

The Western example was also useful in developing mass anti-tobacco campaigns in Poland. The American Cancer Society's "Great American Smokeout" was the inspiration for the "Great Polish Smokeout", organised since 1991, and adapted to the local context. The winners of the competition organised as part of the smokeout won a private audience with Pope John Paul II. The action was a great success, according to Witold Zatoński, with almost four million Poles declaring that they quit thanks to the "Great Polish Smokeout I" [15]. This was a major achievement in a country which in the late 1980s had one of the highest smoking prevalence rates in the world. Since the early 1990s the sale of tobacco in Poland has plummeted from 100 billion cigarettes to around 40 billion today. At the same time the number of smokers has halved, from 16 to 8 million. Most importantly, although one in four Poles are still smokers, both the prevalence and sale of tobacco continue to decrease.

The tobacco industry combats tobacco control policy precedents tooth and nail, as it is afraid of their diffusion. Countries such as Poland, which benefitted from follow-

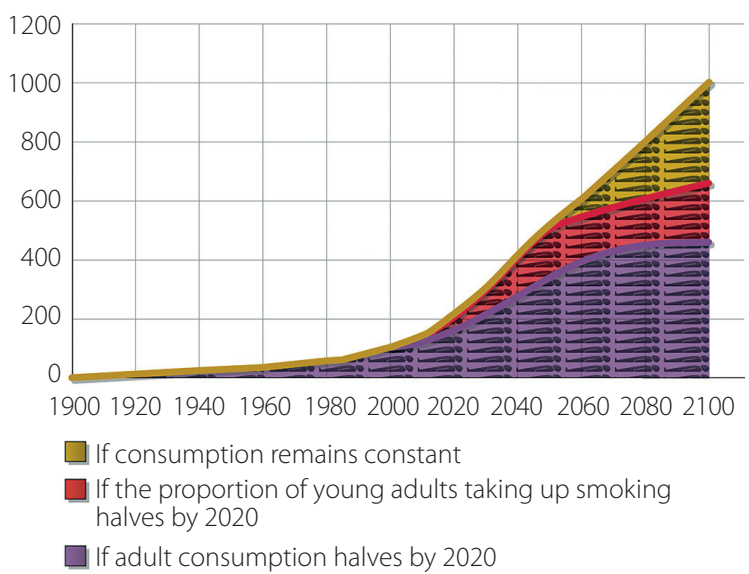

Source: Brandt A, The Cigarette Century: The Rise, Fall and Deadly Persistence of the Product that Defined America. Basic Books, New York 2007.

FIG. 6. Projected total global deaths from cigarette use ing the examples of others, later went on to become models themselves. Mateusz Zatoński brought up the story of the health warning labels introduced by the 1995 law, which at the time were the largest in Europe. This precedent led to policy diffusion, as the European Union decided to follow the Polish example and increase the size of its labels. Allan Brandt mentioned Australia, whose decision to implement plain packaging despite threats from the tobacco industry, is now leading to other countries, such as France or the United Kingdom, adopting the same policy. "What I'm finding", continued Brandt, "is that when the industry's fighting something you can be almost certain that there's good evidence that it's going to hurt them".

The progress in fighting against smoking among children and teenagers was also discussed. Witold Zatoński noted that significant decreases in smoking among children have been recorded in a whole host of countries, from Poland to the US. In Norway, smoking prevalence among 15-year-olds is close to zero. Allan Brandt underlined the importance of this, pointing to evidence that shows that if people get through childhood and adolescents without becoming smokers, their chances to be smokers as adults are impressively reduced. Thus, the decision of some states, such as Hawaii and California, and of some towns, for example Boston, to raise the age legal smoking age to 21 , could be very important.

Greg Connolly closed this section of the discussion by arguing that the progressive declines in smoking that have occurred in many countries in the last decades should be a reminder that tobacco control efforts are going in the right direction. The decline of consumption since 2000 suggests that by the 2030s smoking prevalence in the US will be less than 5\%. The public health community should be careful not to jeopardise these gains by putting excessive faith in new products promising reduced risk. "We don't need them!", concluded Connolly, "Whenever in this nation we have commercialised a psychoactive drug, we've seen addiction rates soar".

\section{ARE WE FACING A "RADICALLY DIFFERENT MOMENT" IN TOBACCO CONTROL?}

The question participants kept returning to throughout the seminar was whether health advocates have found themselves in a different moment in tobacco control history than ever before. Allan Brandt recalled the last great revolution in the history of smoking, when in the 1900s mixed tobacco cigarettes were introduced and promoted by ground-breaking, disruptive changes in modes of marketing. Arguably, a similar revolution is taking place today, in the form of elegant nicotine delivery products using cutting edge technology. "My worry", added Brandt, "and I'm a precautionary principle sort of public health person, is that we don't have the authority to resist this kind of investment, sales, appeal, autonomy and individuality issues, the forces of the market". Rajmund Dąbrowski added that indeed these new prod- 
BOX 1. Meeting participants

\section{Seminar participants}

Allan Brandt is the Amalie Moses Kass Professor of the History of Medicine and Professor of the History of Science at Harvard University. His work focuses on social and ethical aspects of health, disease, medical practices, and global health in the twentieth century.

Gregory Connolly is a Research Professor at the Bouve College of Health Sciences at Northeastern University. His research focuses on tobacco product design, reduced risk tobacco products, global tobacco issues, efficacy of tobacco control interventions and the structure and marketing practices of the tobacco industry.

Rajmund Dąbrowski is a Seventh-day Adventist pastor. He has served as the longtime Director of Communication for the General Conference of the Seventh-day Adventist Church.

Aleksandra Herbeć is a doctoral researcher at the University College London Health Behaviour Research Centre. Her research focuses on the development and mixed-methods evaluation of complex digital interventions for behaviour change and smoking cessation.

Gina Kruse is an Assistant Professor at Harvard Medical School and a clinician investigator in the Division of General Internal Medicine at Massachusetts General Hospital. Her research examines technology-based interventions to improve the delivery of tobacco cessation treatment for patients engaged in healthcare.

Naomi Oreskes is a Professor of the History of Science and Affiliated Professor of Earth and Planetary Sciences at Harvard University. Her research focuses on the earth and environmental sciences, with a particular interest in understanding scientific consensus and dissent.

Nancy Rigotti is a Professor of Medicine at Harvard Medical School and Associate Chief of the Division of General Internal Medicine at Massachusetts General Hospital. She leads a multidisciplinary research group that develops, test, and disseminates interventions for smoking cessation across outpatient and inpatient settings.

Michał Stokłosa is a Senior Economist, Taxation and Health within the Economic and Health Policy Research program at the American Cancer Society. His research focuses on the economics of tobacco control, including tax policy, global illicit cigarette trade, and tobacco industry interference in tobacco control efforts.

Mateusz Zatoński is a doctoral researcher at the London School of Hygiene and Tropical Medicine. His research fo- cuses on the history of anti-tobacco advocacy and tobacco policymaking in Central and Eastern Europe.

Witold Zatoński is the Founder and President of the Health Promotion Foundation, based in Warsaw, Poland. He has launched numerous health campaigns in Central and Eastern Europe. His most recent research examines health inequalities between eastern and western parts of the European Union.

\section{The following scholars participated in the dinner discussion} the day before the seminar:

Grieve Chelwa is a post-doctoral fellow at the Center for African Studies at Harvard University. His doctoral research focused on the economics of tobacco control in South Africa, Uganda and Zambia.

Richard Daynard is a University Distinguished Professor of Law at the Northeastern University School of Law. He is a key leader of the movement to establish the legal responsibility of the tobacco industry for tobacco-induced death, disease and disability.

Sara Kalkhoran is an Instructor in Medicine at Harvard Medical School and a clinician investigator in the Division of General Internal Medicine at Massachusetts General Hospital. Her research interests are in reducing the health risks associated with the use of tobacco products, including emerging products such as electronic cigarettes.

Howard Koh is the Harvey V. Fineberg Professor of the Practice of Public Health Leadership at the Harvard T. H. Chan School of Public Health and the Harvard Kennedy School. Most recently, between 2009 and 2014, he has served as the 14th Assistant Secretary for Health for the U.S. Department of Health and Human Services.

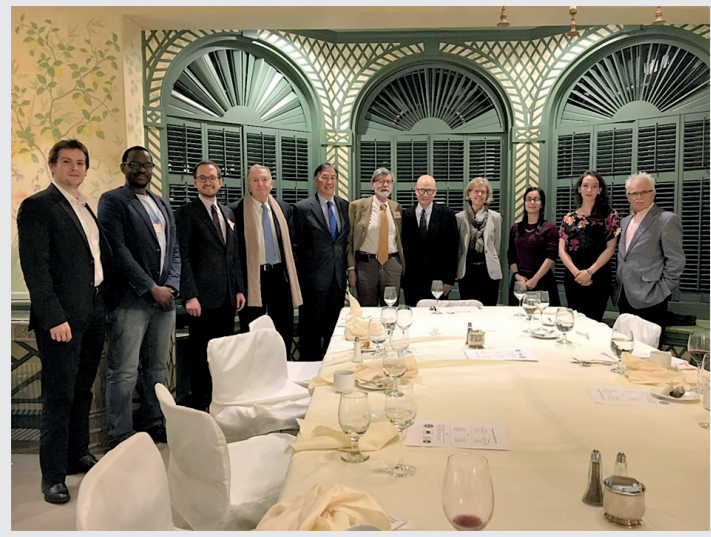

Participants of dinner discussion. From left to right: Mateusz Zatoński, Grieve Chelwa, Michał Stokłosa, Witold Zatoński, Howard Koh, Richard Daynard, Allan Brandt, Nancy Rigotti, Sara Kalkhoran, Aleksandra Herbeć, Rajmund Dąbrowski 
ucts, including e-cigarettes, can be described as "wonderful' in the sense of 'creating wonder, going beyond our understanding".

A number of reasons were outlined by Allan Brandt for why we might be facing a disruptive moment in tobacco control. The first are the changes in systems for nicotine delivery, discussed in depth during the seminar. The second is smokers themselves, who are very different to those who were quitting in the 1980s and 1990s. Those who come to cessation clinics today, pointed out Brandt, have already tried quitting many times and failed. Those who could quit more easily have already done so. Only the most persistent continue to smoke, and they must be approached differently than the "historical smoker" we were used to. Finally, the evaluation of new nicotine delivery products, such as e-cigarettes, might be much more difficult than was the case with combustible cigarettes, for example because of widespread dual use, or existing comorbidities. Nancy Rigotti, however, noted that some evidence suggests the hardening of the smoking population is not as prevalent as is widely assumed, though she admitted that more smokers now have comorbid psychiatric diseases than was the case historically. More of today's smokers are also poor, and quitting smoking is not their primary concern. However, today's smokers also smoke less than before. "So in some ways they're harder', summarised Rigotti, 'in other ways there are still young people starting to smoke [...] and they're probably earlier in the addiction cycle, and maybe those are more like the people 40 years ago who could quit more easily". She emphasised that in this situation it is important to acknowledge that there are different types of smokers for whom different types of treatments need to be tailored.

Allan Brandt closed the discussion by expressing concern that 50 years of progress in tobacco control could be undone in the next 50 years. Even if smoking rates remain unchanged, tobacco will lead to a billion deaths by 2100 . The challenge for the tobacco control field is to promote things we know actually work, while at the same time developing a sharper research agenda to understand the new technologies and changing cultures of smoking.

\section{DISCLAIMER}

The authors alone are responsible for the views expressed in this article, and they do not necessarily represent the views, decisions or policies of the institutions with which they are affiliated.

\section{ACKNOWLEDGEMENTS}

MZ would like to acknowledge the ESR C Bloomsbury DTC for the Social Sciences, which is the funder of the author's $\mathrm{PhD}$ research project on tobacco control history in Poland, as part of which this seminar was organised. Sole responsibility for this research lies with the authors.

\section{ACKNOWLEDGEMENTS FROM EDITORS}

Editors would like to acknowledge the invaluable assistance of Mr. Scott Thompson in the preparation of the final version of this article.

\section{DISCLOSURE}

Authors report no conflict of interest.

\section{References}

1. Marlatt GA. Harm reduction: come as you are. Addict Behav 1996; 21: 779-788.

2. Kilmer B, MacCoun RJ. How medical marijuana smoothed the transition to marijuana legalization in the United States. Annu Rev Law Soc Sci 2017; 13.

3. Morain SR, Winickoff JP, Mello MM. Have Tobacco 21 Laws Come of Age? N Engl J Med 2016; 374: 1601-1604.

4. Royal College of Physicians. Nicotine without smoke: Tobacco harm reduction. RCP, London 2016.

5. O'Brien B, Knight-West O, Walker N, et al. E-cigarettes versus NRT for smoking reduction or cessation in people with mental illness: secondary analysis of data from the ASCEND trial. Tob Induc Dis 2015; 13: 5.

6. Begh R, Lindson-Hawley N, Aveyard P. Does reduced smoking if you can't stop make any difference? BMC Med 2015; 13: 257.

7. O'Connel D. Philip Morris could stop making conventional cigarettes. BBC News, 13 September 2016. Available from: http://www. bbc.com/news/business-38152297. (accessed: 15 March 2017).

8. Murray RL, Bauld L, Hackshaw LE, et al. Improving access to smoking cessation services for disadvantaged groups: a systematic review. J Public Health (Oxf) 2009; 31: 258-277.

9. Anthenelli RM, Benowitz NL, West R, et al. Neuropsychiatric safety and efficacy of varenicline, bupropion, and nicotine patch in smokers with and without psychiatric disorders (EAGLES): a double-blind, randomised, placebo-controlled clinical trial. Lancet 2016; 387: 2507-2520.

10. Tutka P, Zatonski W. Cytisine for the treatment of nicotine addiction: from a molecule to therapeutic efficacy. Pharmacol Rep 2006; 58: 777-798.

11. Stokłosa M, Drope J, Zatoński M, et al. Towards improved public health: affecting alcohol and tobacco affordability and consumption in Poland through taxations. J Health Inequal 2016; 2: 101-104.

12. Levinson AH. Where the U.S. tobacco epidemic still rages: most remaining smokers have lower socioeconomic status. J Health Care Poor Underserved 2017; 27: 100-107.

13. GBD 2015 Tobacco Collaborators. Smoking prevalence and attributable disease burden in 195 countries and territories, 1990-2015: a systematic analysis from the Global Burden of Disease Study 2015. Lancet 2017; 389: 1885-1906.

14. Zatoński M. Poland's anti-tobacco advocacy - a historical outline. J Health Inequal 2016; 2: 26-31.

15. Jassem J, Przewoźniak K, Zatoński W. Tobacco control in Poland - successes and challenges. Transl Lung Cancer Res 2014; 3 : 280-285. 